\title{
Molecular insight into the docking of lysophosphatidylethanolamine (lysoPE)-22:6 interaction with GPR119: Acute exposure to an electric field induces changes in human plasma lysoPE-22:6 and lysoPE-20:4 levels
}

\author{
Yuzo Nakagawa-Yagi ${ }^{1 *}$, Hiroyuki Hara ${ }^{1}$, Hiroki Nakanishi ${ }^{2}$, Chisato Kanai ${ }^{3}$ and Akikuni Hara ${ }^{1}$ \\ ${ }^{1}$ Hakuju Institute for Health Science Co., Ltd., 37-5 Tomigaya 1-chome, Shibuya-ku, Tokyo 151-0063, Japan \\ ${ }^{2}$ Akita Lipid Technologies LLC, 1-2 Nukazuka, Yanagida, Akita, Akita 010-0825, Japan \\ ${ }^{3}$ Kyoto Constella Technologies Co., Ltd., Kyozome Kaikan, 481Tourouyama-cho, Nakagyo-ku, Kyoto 604-8225, Japan
}

\begin{abstract}
Medical treatment using high-voltage electric potential (HELP) devices to generate an electric field (EF) is an alternative therapy commonly used in Japan. However, the underlying mechanisms of the potential health benefits of this therapy are not fully understood. Therefore, we investigated the lysophosphatidylethanolamine (lysoPE) and lysophosphatidylinositol (lysoPI) levels using selected reaction monitoring (SRM) analysis in plasma samples obtained from healthy human subjects before and after exposure to a single HELP exposure (9 kV/electrode + $9 \mathrm{kV} /$ electrode, $30 \mathrm{~min})$. LysoPE-22:6 and lysoPE-20:4 were significantly upregulated after HELP exposure. However, there were no effects on the levels of phosphatidylethanolamine (PE) and phosphatidylinositol (PI), lysoPI, or other lysoPE species. LysoPE is known to activate G protein-coupled receptor 119 (GPR119). No X-ray crystal structure has been reported for GPR119; thus we examined the in silico docking simulation of lysoPE-22:6 with GPR119 using a homology model. LysoPE-22:6 showed strong interaction energy of -10.603 kcal/mol. Our findings provide new insight into the molecular mechanisms of the health benefits of EF therapy.
\end{abstract}

\begin{abstract}
Abbreviations: AR231453:N-(2-fluoro-4-methylsulfonylphenyl)5-nitro-6-[4-(3-propan-2-yl-1,2,4-oxadiazol-5-yl)piperidin-1-yl] pyrimidin-4-amine; EF: electric field; GLP-1: glucagon-like peptide-1; GPR119: G protein-coupled receptor 119; HELP: high-voltage electric potential; lysoPC: lysophosphatidylcholine; lysoPC-22:4: (2-\{[(2R)-3-[(7Z,10Z,13Z,16Z)-docosa-7,10,13,16-tetraenoyloxy]2-hydropropyl phosphonato]oxy\}ethyl)trimethylazanium; lysoPE: lysophosphatidylethanolamine; lysoPE-22:6: (2-aminoethoxy)[(2R)2-[(4Z, 7Z, 10Z, 13Z, 16Z, 19Z)-docosa-4,7,10,13,16-hexaenoyloxy]3-hydroxypropoxy]phosphinic acid; lysoPE-20:4: (2-aminoethoxy) [(2R)-2-hydroxy-3-[(5Z, 8Z, 11Z, 14Z)-icosa-5,8,11,14-tetraenoyloxy] propoxy]phosphinic acid; lysoPI: lysophosphatidylinositol; OEA: oleoylethanolamine; PE: phosphatidylethanolamine; PI: phosphatidylinositol; PLA $\mathrm{P}_{2}$ : phospholipase $\mathrm{A}_{2}$; PLC: phospholipase C; PLD: phospholipase D; or SRM: selected reaction monitoring.
\end{abstract}

\section{Introduction}

A therapeutic device to expose the human body to high-voltage electric potential (HELP) has been approved by the Ministry of Health, Labour and Welfare in Japan [1-16]. High-voltage electric field (EF) therapy is reported to be an effective treatment for stiff shoulders, headache, insomnia, and chronic constipation [1-17]. Sincethe discovery of EF therapy more than 80 years ago, its molecular mechanisms of health benefits have remained a mystery. Altogether, the results of these studies suggest that HELP exposure may present an alternative therapy for several conditions, although the underlying mechanisms of action remain elusive. Our previous attempts to find a HELP exposure-induced biomarker using plasma metabolomics have led to the detection of lipid-derived signaling molecules such as oleoylethanolamine (OEA), cis-8,11,14-eicosatrienoic acid, cis-4,7,10,13,16,19-docosahexaenoic acid, linoleic acid, 9-hydroxyoctadecadienoic acid (9-HODE), 13HODE, 13-hydroperoxy-octadecadienoic acid (13-HpODE), and 3-hydroxybutyrate (3-HBA), a lipid-derived histone deacetylase inhibitor [12-16]. Endogenous lipid-derived signaling molecules have been suggested as candidate molecules representing the interface between symptoms and electroceutical target proteins [12-16]. A recent study by Hansen et al. reported that GPR119-mediated cyclic AMP accumulation is potentiated by OEA, 2-oleoyl glycerol (2AG), or lysoPE in transiently transfected COS-7 cells [18]. In our previous study, we observed HELP exposure-induced upregulation of lysophosphatidylcholine (lysoPC)-22:4 levels in the plasma of healthy individuals [16]. Therefore, we hypothesized that the increased plasma

Correspondence to: Yuzo Nakagawa-Yagi, Ph.D, Hakuju Institute for Health Science Co., Ltd., 37-5 Tomigaya 1-chome, Shibuya-ku, Tokyo 151-0063, Japan

Key words: lysophosphatidylethanolamine, lysoPE-22:6, GPR119, electric field therapy

Received: September 13, 2017; Accepted: September 19, 2017; Published: September 22, 2017 
lysoPC-22:4 levels following EF exposure might be linked to changes in lysoPE or lysoPI species. Thus, we investigated the levels of lysoPE and lysoPI using SRM analysis in plasma samples obtained from healthy subjects before and after exposure to a single HELP stimulation. Here we report that plasma lysoPE-22:6 and lysoPE-20:4 levels can be upregulated by HELP exposure $(9 \mathrm{kV} /$ electrode $+9 \mathrm{kV} /$ electrode, 30 min). We also conducted a binding study using AutoDock Vina docking software to explore the interactions of lysoPE-22:6 or lysoPE-20:4 with a homology model of GPR119 using the template structure (PDB ID 4QKX).

\section{Materials and methods}

\section{EF exposure}

The system used for EF exposure has previously been described [12-17]. The EF system was equipped with a transformer, a seat, and two insulator-covered electrodes. One electrode was placed on a floor plate on which the subject's feet were located and the other electrode was placed above the subject's head. EF generated by the HELP apparatus (Healthtron PRO-18T; Hakuju Institute for Health Science Co., Ltd., Tokyo, Japan) was uniformly created by transforming a 50 $\mathrm{Hz}$ alternating current at $18 \mathrm{kV}$ ( $9 \mathrm{kV} /$ electrode $+9 \mathrm{kV} /$ electrode $)$. The safety of this system for human use was established by the Japanese government in 1963.

\section{Subjects}

Fifty healthy adults [ 21 males and 29 females; mean age, $46.5 \pm 0.9$ years; mean body mass index (BMI), $21.9 \pm 0.4 \mathrm{~kg} / \mathrm{m}^{2}$ ] participated in experiment 1 (exposure condition: $9 \mathrm{kV} /$ electrode $+9 \mathrm{kV} / \mathrm{electrode}$, $30 \mathrm{~min}$ ). Twenty-five healthy adults [ 9 males and 16 females; mean age, $46.1 \pm 1.1$ years; mean body mass index (BMI), $\left.22.2 \pm 0.5 \mathrm{~kg} / \mathrm{m}^{2}\right]$ participated in experiment 2 (exposure condition: $9 \mathrm{kV} /$ electrode +9 $\mathrm{kV} /$ electrode, $30 \mathrm{~min}$ ). All experiments were performed in the morning and all participants signed an informed consent form after receiving verbal and written information about the study. All experiments were conducted in accordance with the Declaration of Helsinki and the study protocol was approved by the human ethics committee of Hakuju Institute for Health Science Co., Ltd. (Tokyo, Japan).

\section{Plasma preparation}

Blood samples were collected in vacutainer tubes coated with ethylenediaminetetraacetic acid (VP-NA070K; Terumo Corporation, Tokyo, Japan) and immediately centrifuged at $800 \mathrm{xg}$ for $5 \mathrm{~min}$ to separate the plasma from other cellular materials. Plasma was then transferred to a fresh Eppendorf tube and stored at $-80^{\circ} \mathrm{C}$ until processing.

\section{Phospholipid preparation}

Comprehensive analysis of phospholipids was done essentially in the same manner as described previously $[16,19-20]$. Briefly, total phospholipids were extracted from the plasma using the Bligh-Dyer method [21]. Aliquots of the lower/organic phase were evaporated to dryness under $\mathrm{N}_{2}$ and the residue was dissolved in methanol for LC-MS/MS measurements of phosphatidylethanolamine (PE) and phosphatidylinositol (PI).

\section{Mass spectrometric analyzes}

LC-electrospray ionization-MS/MS analysis was performed using an UltiMate 3000 LC system (Thermo Fisher Scientific, Waltham, MA, USA) equipped with an HTC PAL autosampler (CTC Analytics AG, Lake Elmo, MN, USA). A $10 \mu \mathrm{L}$ aliquot of the lipid samples was injected and the lipids were separated on a Waters X-Bridge C18 $(3.5 \mu \mathrm{m}, 150$ $\mathrm{mm}$ x $1.0 \mathrm{~mm}$ i.d., Waters Corporation, Milford, MA, USA) at room temperature $\left(25^{\circ} \mathrm{C}\right)$ using a gradient solvent system as follows: mobile phase A [isopropanol/methanol/water (5:1:4, v/v/v) supplemented with $5 \mathrm{mM}$ ammonium formate and $0.05 \%$ ammonium hydroxide] / mobile phase B (isopropanol supplemeted with $5 \mathrm{mM}$ ammpnium formate and $0.05 \%$ ammonium hydroxide) ratios of $70 \% / 30 \%(0$ $\mathrm{min}), 50 \% / 50 \%(2 \mathrm{~min}), 20 \% / 80 \%$ (13 $\mathrm{min}), 5 \% / 95 \%$ (15-30 $\mathrm{min}$ ), $95 \% / 5 \%(31-35 \mathrm{~min})$, and $70 \% / 30 \%(35-45 \mathrm{~min})$. Flow rate was 20 $\mu \mathrm{L} / \mathrm{min}$. Phospholipid species were measured by SRM in positive ion mode with a TSQ Vantage AM mass spectrometer (Thermo Fisher Scientific, Waltham, MA, USA). The characteristic fragments of individual phospholipids were detected by the product ion scan (MS/ MS mode). Chromatographic peak areas were used for comparative quantitation of each molecular species (e.g., 38:6, 40:6) in a given class of the phospholipids. The specific detection of individual lipids was performed using multiple reaction monitoring. Multiple reaction monitoring $(\mathrm{MRM}) \mathrm{m} / \mathrm{z}$ transitions were : lysoPE-22:6 = 526.3/385.3; or lysoPE-20:4 = 502.3/361.3.

\section{Homology modeling and docking simulation}

We used Q8TDV5.fasta registered in UniProt for sequence information of hGPR119 (GP119: glucose-dependent insulinotropic receptor). There were eleven PDB (Protein Data Bank)-IDs of the 3D structure (4LDE, 4QKX, 2YDO, 5G53, 4MQS, 3P0G, 3PDS, 4IAR, 4IAQ, 4IB4, 5TVN) that could be used as a template structure. From the docking calculation between OEA (an endogenous GPR119 agonist) and each homology model, a model (based on $4 \mathrm{QKX}$ ) with the best docking score was chosen [22-23]. A template structure (PDB ID Code $4 \mathrm{QKX}$ ) beta-2-adrenergic receptor-T4 lysozyme fusion protein was modeled as target protein hGPR119. The docking study of lysoPE-22:6 binding to the target protein of the hGPR119 model structure was performed using AutoDock Vina docking software (Dr. Oleg Trott, The Scripps Research Institute, CA, USA) [24]. The docking experiment was performed five times and yielded 100 candidate conformations.

\section{Statistical analysis}

Data were analyzed using Welch's $t$-test. A probability $(p)$ value $<$ 0.05 was considered statistically significant.

\section{Results}

\section{Effect of HELP exposure on lysoPE and PE in plasma from healthy humans}

We assessed lysoPE in the plasma obtained from 50 healthy participants using SRM analysis (Figure $1 \mathrm{a}-\mathrm{b}$ and Figure 2). HELP exposure $(9 \mathrm{kV} /$ electrode $+9 \mathrm{kV} /$ electrode, $30 \mathrm{~min})$ resulted in significantly higher plasma lysoPE-22:6 and lysoPE-20:4 levels than pre-exposure levels (lysoPE-22:6: 1.48 -fold; $p=0.032$; lysoPE-20:4: 1.42 -fold, $p=0.038$ ). Under these conditions, HELP exposure did not affect the lysoPE-16:0, lysoPE-18:0, lysoPE-18:1, lysoPE-18:2, lysoPE-20:5, or lysoPE-22:5 levels (Figure 2).

We assessed PE in the plasma obtained from 50 healthy participants using SRM analysis. HELP exposure (9 kV/electrode $+9 \mathrm{kV} /$ electrode, 30 min) did not affect the PE-34:1, PE-34:2, PE-36:1, PE-36:2, PE-36:3, PE36:4, PE-38:4, PE-38:5, PE-38:6, PE-40:4, PE:40:5, or PE-40:6 levels (Figure 3).

\section{Effect of HELP exposure on lysoPI and PI in plasma from healthy humans}

We assessed lysoPI in the plasma obtained from 25 healthy participants using SRM analysis. HELP exposure $(9 \mathrm{kV} /$ electrode + 


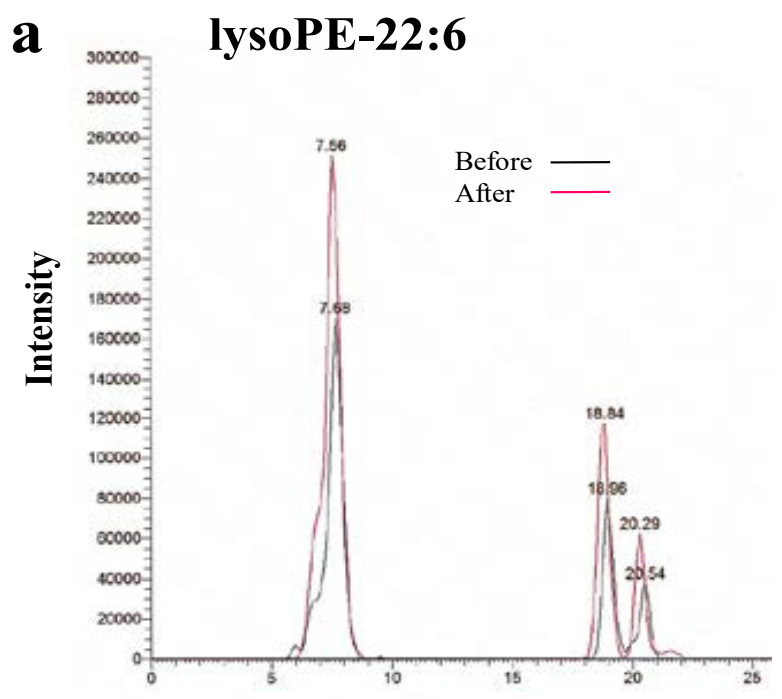

Time (min) b lysoPE-20:4

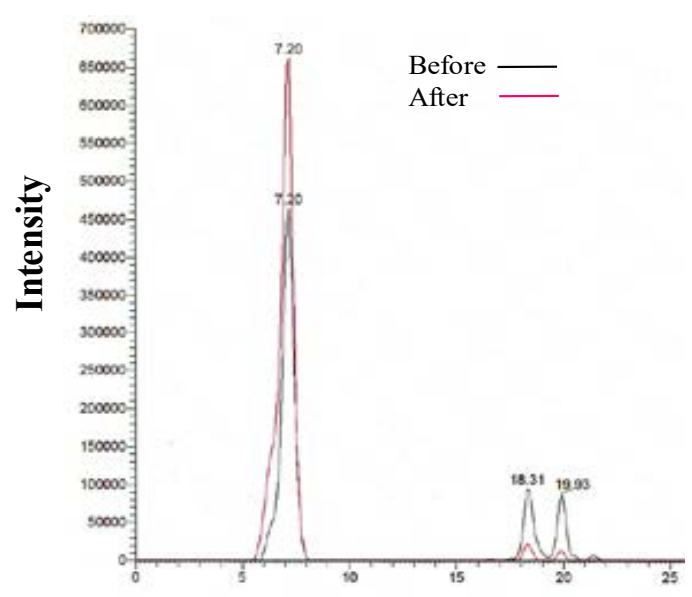

Time (min)

Figure 1. Lipidomic analysis of the plasma of healthy individuals before and after HELP exposure for $30 \mathrm{~min}$.

(a) Typical lysoPE-22:6 peak in the plasma of healthy humans. LysoPE-22:6 was detected by SRM analysis. (b) Typical lysoPE-20:4 peak in the plasma of healthy humans. LysoPE-20:4 was detected by SRM analysis.

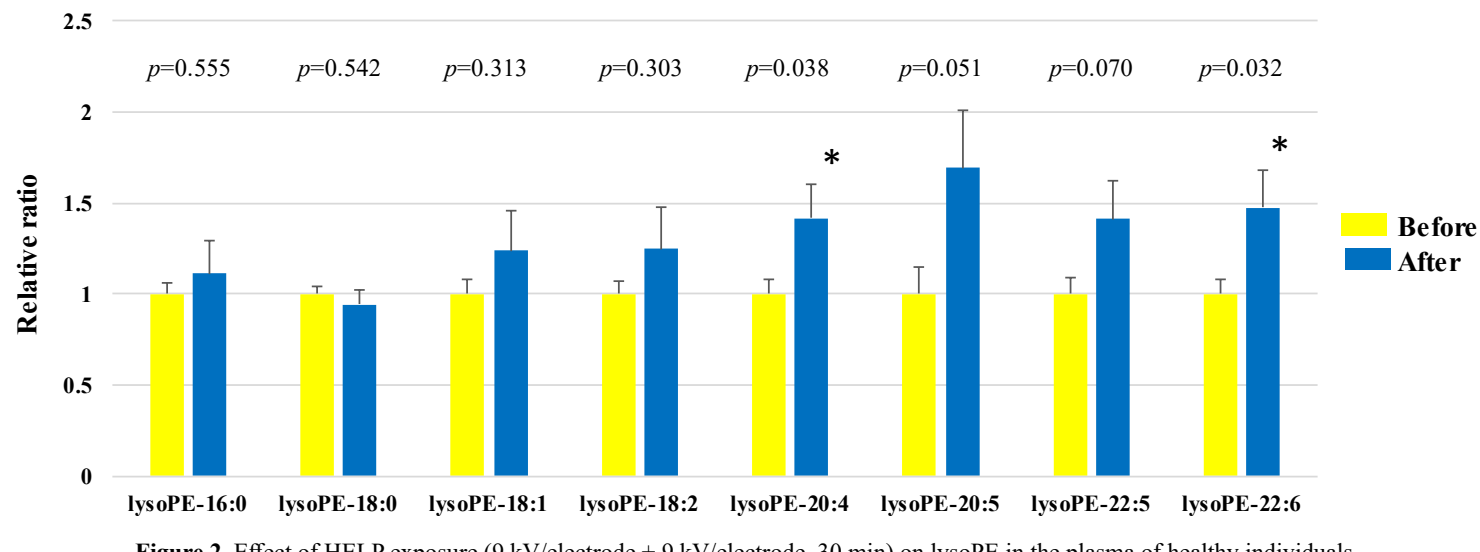

Figure 2. Effect of HELP exposure $(9 \mathrm{kV} /$ electrode $+9 \mathrm{kV} /$ electrode, $30 \mathrm{~min})$ on lysoPE in the plasma of healthy individuals. Relative ratio (after/before) of lysoPE in plasma before and after EF exposure. Results are presented as mean SEM $(\mathrm{n}=50) .{ }^{*} p<0.05$ compared with before.

1.5

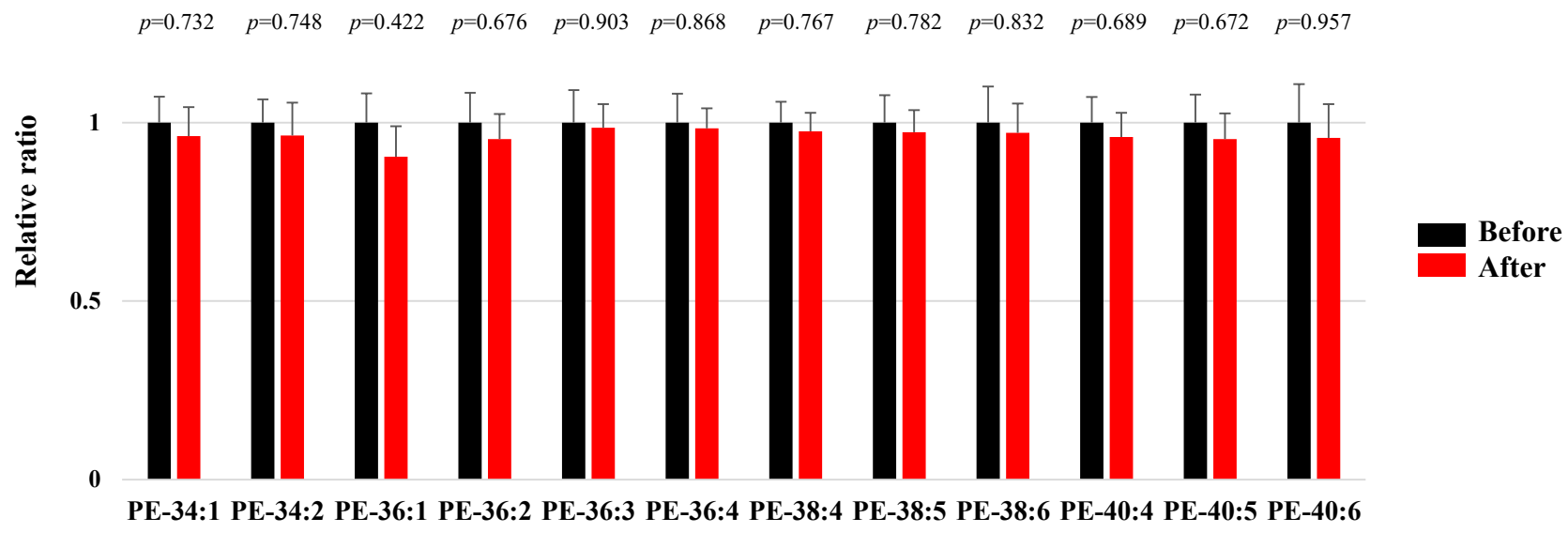

Figure 3. Effect of HELP exposure $(9 \mathrm{kV} / \mathrm{electrode}+9 \mathrm{kV} / \mathrm{electrode}, 30 \mathrm{~min})$ on PE in the plasma of healthy individuals.

Relative ratio (after/before) of PE in plasma before and after EF exposure. Results are presented as mean SEM $(\mathrm{n}=50)$. 
$9 \mathrm{kV} /$ electrode, $30 \mathrm{~min}$ ) did not affect the lysoPI-16:0, lysoPI-18:0, lysoPI-18:1, or lysoPI-20:4 levels (Figure 4).

We assessed PI in the plasma obtained from 25 healthy participants using SRM analysis. HELP exposure $(9 \mathrm{kV} /$ electrode $+9 \mathrm{kV} /$ electrode, $30 \mathrm{~min}$ ) did not affect the PI-32:0, PI-32:1, PI-34:0, PI-34:1, PI-34:2, PI-36:0, PI-36:1, PI-36:2, PI-36:3, PI-36:4, PI-38:3, PI-38:4, PI-38:5, PI38:6, PI-40:4, PI-40:5, or PI-40:6 levels (Figure 5).

\section{Docking of lysoPE-22:6 and lysoPE-20:4 on GPR119}

LysoPE-18:1 is known to stimulate the accumulation of cyclic AMP by activation of human GPR119 in transiently transfected COS-7 cells [25]. Therefore, we hypothesized that increased plasma lysoPE-22:6 or lysoPE-20:4 levels after HELP exposure may be linked to its activation as an endogenous agonist of GPR119. We examined the in silico docking of lysoPE-22:6, lysoPE-20:4, lysoPC-22:4, or a well-known GPR119 agonist, AR231453, in the active site of GPR119 using AutoDock Vina software [22-23, 26]. Docking results showed that lysoPE-22:6 has good binding energy of $-10.603 \mathrm{kcal} / \mathrm{mol}$ (Table 1). LysoPE-22:6 formed hydrogen bonds with Gln65, Arg81, Cys155, and Ser156 (Table 1, Figure 6a). In addition, lysoPE-20:4 showed strong interaction energy of $-9.756 \mathrm{kcal} / \mathrm{mol}$ (Table 1). LysoPE-20:4 formed hydrogen bonds with Ser156, Phe157, and Glu261 (Table 1, Figure 6b). A similar docking score was obtained using lysoPC-22:4 instead of lysoPE-20:4 (Table 1). LysoPC-22:4 formed hydrogen bonds with Gln154, and Phe157 (Table 1, Figure 6c). Under these conditions, the potent GPR119 agonist, AR231453, showed good binding energy ( $-12.409 \mathrm{kcal} / \mathrm{mol}$ ) (Table 1). AR231453 formed hydrogen bonds with Arg81, Phe157, and Arg262 (Table 1, Figure 6d).

\section{Discussion}

In this study, we showed that lysoPE-22:6 and lysoPE-20:4 are sensitive to acute EF exposure in healthy human subjects. Notably, the absence of a lysoPI response indicates that lysoPE-22:6 and LysoPE-20:4 responses are not adverse nonspecific actions on the membrane lipids. The molecular mechanisms of the changes in lysoPE-22:6 and lysoPE-20:4 levels following EF exposure are complex and can be interpreted in several ways. We have previously shown an acute EF exposure $(9 \mathrm{kV} /$ electrode $+9 \mathrm{kV} /$ electrode, 30 min)-induced increase of approximately 1.51-fold and 1.41-fold for cis-4,7,10,13,16,19-docosahexaenoic acid (FA-22:6) and arachidonic

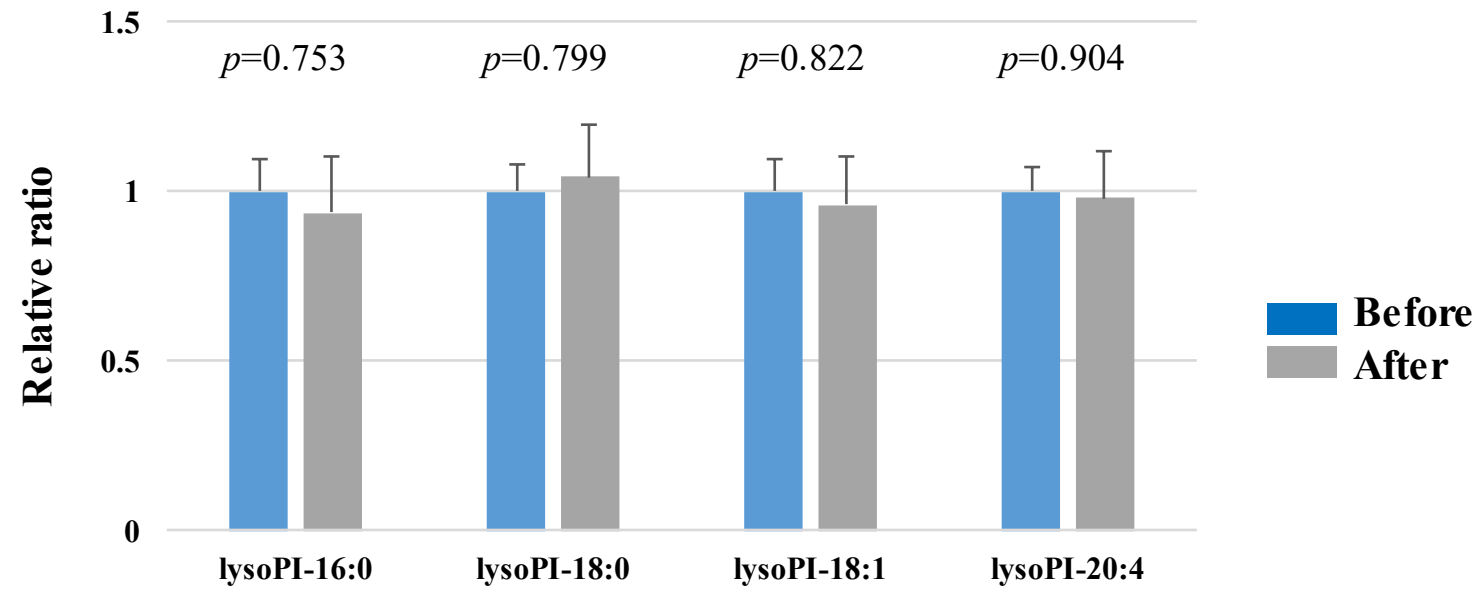

Figure 4. Effect of HELP exposure $(9 \mathrm{kV} / \mathrm{electrode}+9 \mathrm{kV} / \mathrm{electrode}, 30 \mathrm{~min})$ on lysoPI in the plasma of healthy individuals. Relative ratio (after/before) of lysoPI in plasma before and after EF exposure. Results are presented as mean SEM $(n=25)$.

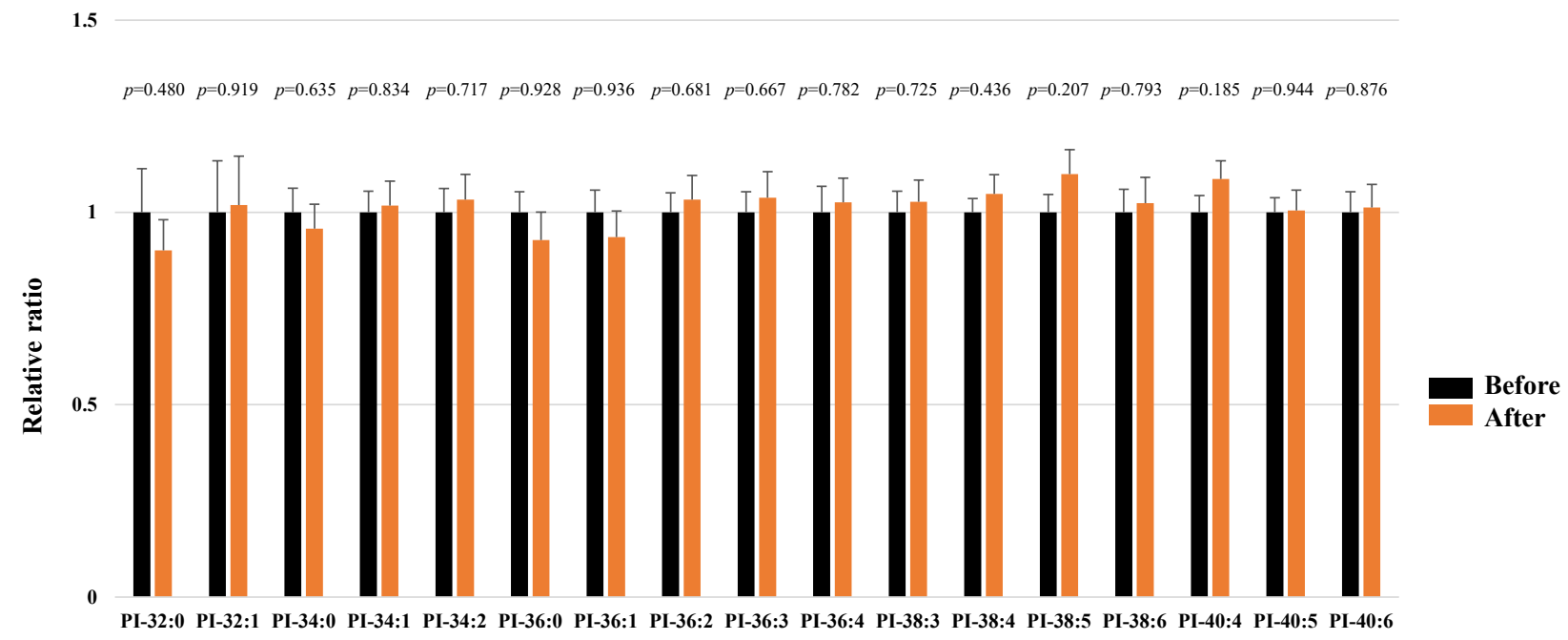

Figure 5. Effect of HELP exposure $(9 \mathrm{kV} /$ electrode $+9 \mathrm{kV} /$ electrode, $30 \mathrm{~min})$ on PI in the plasma of healthy individuals.

Relative ratio (after/before) of PE in plasma before and after EF exposure. Results are presented as mean SEM $(\mathrm{n}=25)$. 

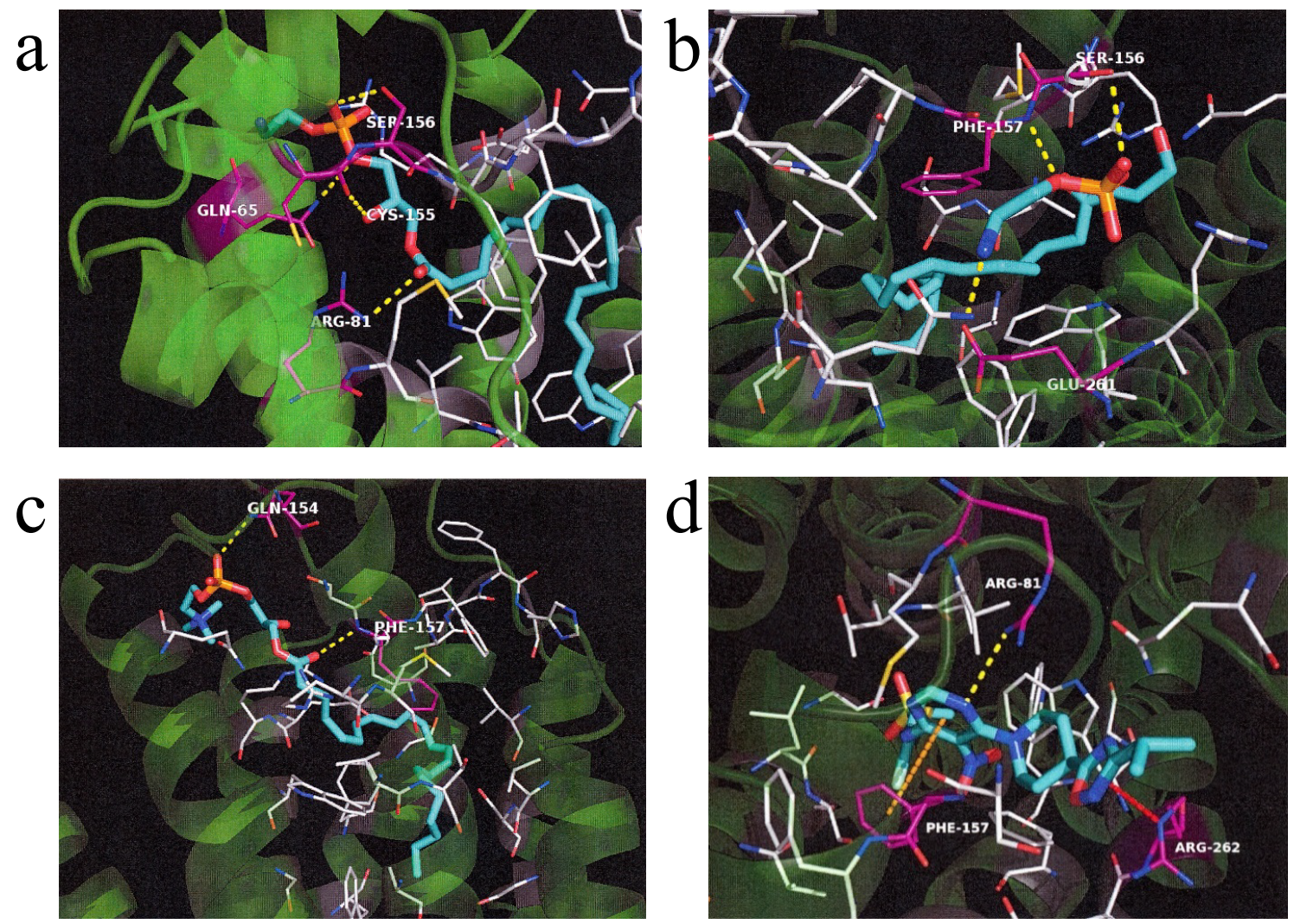

Figure 6. In silico molecular docking of lysoPE-22:6, lysoPE-20:4, lysoPC-22:4, or AR231453 with GPR119.

(a) Binding mode of lysoPE-22:6 in homology modeling of GPR119. Cyan represents lysoPE-22:6 and white represents the amino acid making up the pocket. The yellow dashed line indicates hydrogen bonding, and the hydrogen bonding partner amino acid is represented by magenta. The phosphoryl ester moiety of lysoPE-22:6 forms a hydrogen bond with the side chain of Glu65 and Ser156. The carboxyl of the ester moiety of lysoPE-22:6 forms a hydrogen bond with the carboxyl in the main chain of Cys155. The carboxyl of the ester moiety of lysoPE-22:6 forms a hydrogen bond with the side chain of Arg81. (b) Binding mode of lysoPE-20:4 in homology modeling of GPR119. Cyan represents lysoPE-20:4 and white represents the amino acid making up the pocket. The yellow dashed line indicates hydrogen bonding and the hydrogen bonding partner amino acid is represented by magenta. The phosphoryl ester moiety of lysoPE-20:4 forms a hydrogen bond with the hydroxyl group in the side chain of Ser156 and the nitrogen in main chain of Phe157. The primary amine at the tip of lysoPE-20:4 forms a hydrogen bond with the carboxylic acid moiety of Glu261. (c) Binding mode of lysoPC-22:4 in homology modeling of GPR119. Cyan represents lysoPC-22:4 and white represents the amino acid making up the pocket. The yellow dashed line indicates hydrogen bonding and the hydrogen bonding partner amino acid is represented by magenta. The phosphoryl ester moiety of lysoPC-22:4 forms a hydrogen bond with the side chain of Gln154. The ester moiety of lysoPC-22:4 forms a hydrogen bond with the nitrogen in the main chain of Phe157. (d) Binding mode of AR231453 in homology modeling of GPR119. Cyan represents AR231453 and white represents the amino acid making up the pocket. The yellow dashed line indicates hydrogen bonding, and the hydrogen bonding partner amino acid is represented by magenta. The orange dashed line indicates $\pi$ - $\pi$ interaction, while the red dashed line indicates cation- $\pi$ interaction. The nitrogen atom in the pyrimidine ring of AR231453 forms a hydrogen bond with the guanidyl group of Arg 81 and the benzene ring of Phe157 interacts with the pyrimidine ring by $\pi-\pi$. The oxadiazole group of AR 231453 and the guanidyl group of Arg262 interacts with cation- $\pi$.

Table 1. Docking score and key interacting residues of GPR119.

\begin{tabular}{|l|l|l|}
\hline Ligand & Docking score (kcal/mol) & Interactive residues \\
\hline LysoPE-22:6 & -10.603 & Gln-65, Arg-81, Cys-155, and Ser-156 \\
\hline LysoPE-20:4 & -9.756 & Ser-156, Phe-157, and Glu-261 \\
\hline LysoPC-22:4 & -9.823 & Gln-154, and Phe-157 \\
\hline AR231453 & -12.409 & Arg-81, Phe-157, and Arg-262 \\
\hline
\end{tabular}

acid (FA-20:4), respectively [12]. Notably, Thuren et al. reported that phospholipase $\mathrm{A}_{2}\left(\mathrm{PLA}_{2}\right)$-catalyzed hydrolysis is elevated by EF [27]. $\mathrm{PLA}_{2}$ enzymes hydrolyze the ester of glycerophopholipids to release lysophospholipids and a free polyunsaturated fatty acid [28]. We have shown that PLD-mediated breakdown product ethanolamine and PLCmediated breakdown product ethanolamine phosphate do not change by EF exposure ( $9 \mathrm{kV} /$ electrode $+9 \mathrm{kV} /$ electrode, $30 \mathrm{~min})$ [12]. Thus, it is reasonable to speculate that EF exposure upregulates lysoPE-22:6 and lysoPE-20:4 through the activation of PLA. In particular, understanding the nature and molecular regulation of potential anti-inflammatory PLA is important for alleviating the chronic inflammation in various intractable diseases such as rheumatoid arthritis, inflammatory bowel disease, Crohn's disease, and Alzheimer disease [29-32]. We found that OEA induces marked upregulation in group IID secretory PLA 2 expression in human subcutaneous cultured adipocytes [12]. In the future, it will be of interest to identify the sensitive PLA, subtype induced by EF exposure.

Another goal of the present study was to gain insight into the molecular mechanisms of the health benefit provided by EF therapy. Interestingly, Hansen et al. reported that lysoPE-18:1 increases the intracellular concentration of cyclic AMP in GPR119-expressing COS7 cells [25]. Unfortunately, there lysoPE-22:6 and lysoPE-20:4 are not commercially available as pure chemical reagents for pharmacological experiments. Thus, it will take some time for us to investigate the effects of lysoPE-22:6 and lysoPE-20:4 on the accumulation of cyclic AMP levels in HEK293 $\mathrm{T}$ or CHO-K1 cells stably expressing human GPR119. An increasing number of reports on virtual simulation have appeared in the literature [16, 33-34]. In silico molecular docking studies have been used to support the pharmacological results. However, no crystal structure has been reported for GPR119. Thus, we focused on homology modeling of GPR119. In the present study, the docking simulation showed that lysoPE-22:6 or lysoPE-20:4 can bind to GPR119. LysoPE-22:6 binding to GPR119 was found to be stabilized through the formation of hydrogen bonds with Gln65, Arg81, Cys155, 
Nakagawa-Yagi Y (2017) Molecular insight into the docking of lysophosphatidylethanolamine (lysoPE)-22:6 interaction with GPR119: Acute exposure to an electric field induces changes in human plasma lysoPE-22:6 and lysoPE-20:4 levels

and Ser156, and lysoPE-20:4 binding to GPR119 was found to be stabilized through the formation of hydrogen bonds with Ser156, Phe157, and Glu261. Interestingly, a previous study examining binding pockets using a homology model (PDB ID 2RH1) of GPR119 reported hydrogen bonds to Phe157 [35]. In the future, it will be of interest to identify the binding pocket of lysoPE-22:6 and lysoPE-20:4 in human GPR119 using the crystal structure.

We recently found, using human metabolomics and lipidomics, that the increase in plasma OEA and lysoPC-22:4 levels is elicited by EF exposure $[12,16]$. Moreover, we reported that EF exposure induces a transient increase of glucagon-like peptide-1 (GLP-1) levels in plasma [13]. Considerable evidence for GLP-1 secretion induced by endogenous agonists of GPR119 containing OEA, lysoPC, or lysoPE has been obtained from studies of intestinal enteroendocrine L-cells [23, 36-37]. It is thus reasonable to speculate that EF exposure activates GLP-1 secretion through the activation of GPR119. However, it is unclear at the present whether the changes in GLP-1 levels are controlled individually or all together by OEA, lysoPC-22:4, lysoPE-20:4 or lysoPE-22:6.

A recent study showed that GLP-1 can influence brain neuronal activities in the nucleus of the solitary tract via an alternative pathway such as the gut-to-brain-to-periphery axis [38-39]. Campolongo et al. reported that post-training peripheral administration of OEA enhances memory consolidation in spatial learning behavior [40]. These effects of OEA are blocked by infusion of lidocaine into the nucleus of the solitary tract [40]. Interestingly, Yanamoto et al. reported that EF exposure (5h/day for 3 weeks) induces improved performance in the Morris water maze tasks in a mouse model of infarct lesions [41]. Thus, it is reasonable to speculate that $\mathrm{EF}$ exposure facilitates spatial learning and memory function via the gut-to-brain-to-periphery axis. Further studies are needed to clarify the central players in the gut-to-brain-toperiphery axis signaling pathways induced by EF exposure.

In conclusion, acute EF exposure exerted marked effects on plasma lysoPE-22:6 and lysoPE-20:4 levels in healthy subjects, and in silico docking of lysoPE-22:6 and lysoPE-20:4 was observed in the homology model of GPR119. Our findings provide insight into the molecular mechanisms behind the health benefits induced by the HELP device.

\section{Competing interests}

$\mathrm{YN}-\mathrm{Y}, \mathrm{HH}$, and $\mathrm{AH}$ are employed by Hakuju Institute for Health Science Co., Ltd.; HN is employed by Akita Lipid Technologies LLC.; and CK is employed by Kyoto Constella Techologies Co., Ltd. All other authors have no competing interests.

\section{Authors' contributions}

YN-Y designed and supervised the research, and wrote the manuscript. HN performed SRM. CK performed the molecular modeling. $\mathrm{YN}-\mathrm{Y}, \mathrm{HH}$, and $\mathrm{AH}$ performed the biophysical experiments. All authors have read and approved the final version of the manuscript.

\section{Acknowledgment}

We thank Dr. Makoto Kikuchi (Professor Emeritus, National Defense Medical College, Japan) for encouragement.

\section{References}

1. Hara H (1961) On the effect of AC. electrostatic high voltage potential load upon the blood-electrolytes (in Japanese). Niigata Medical J 75: 265-273.

2. Ito F, Furuya K (1981) The effect of high voltage alternating current upon a human body the change of blood pressure, endocrine system and serum lipids (in Japanese). $J$ Jpn Sci Balneol Climatol Phys Med 45: 6-17.
3. Isaka K, Nishimura R, Arase S, Takiwaki H, Osaki K, et al. (1998) Dosimetry and exposure experiments for extremely low frequency high-tension electric field therapy. EMC '98 Rome International Symposium on Electromagnetic Comoatibility D: 204-207.

4. Nawarat S, Iomsai K, Jantanam P, Kauengtip Y (1999) Effects of electrical Healthtron on curing of non-communicable diseases: Case study of Banlad hospital Petchaburi province (in Thai). Region 4 Medical J 18: 139-149.

5. Ito F (2000) The role of electric field therapeutic device (Healthtron) in the therapy of acute low back pain (in Japanese). J Jpn Sci Balneol Climatol Phys Med 63: 127-137.

6. Siripanichgon K, Otrakul A, Suparp J, Sirikulchayanonta C, Charupoonphol P (2000) Clinical observation of Healthtron therapy (in Thai). J Public Health (Bangkok) 30: 19-29.

7. Sirikulchayanonta C, Siripanichgon K, Otrakul A, Suparp J, Charupoonphol P (2001) J Public Health (Bangkok) 31: 63-70.

8. Ito F, Ohsaki K, Takahashi K, Hara H (2005) The effects of electric field therapeutic device (Healthtron) on the stiffness in the neck and shoulder area - changes in subjective symptoms, blood circulation and the autonomic nervous system (in Japanese). J Jpn Sci Balneol Climatol Phys Med 68: 110-121.

9. Zang L, Wang Q, Peng N, Chen W, Li X, et al. (2012) High-voltage electrostatic therapy for chronic sleep disorder in aged patients (in Chinese). Academic J PLA Postgrad Med Sch 33 730-732.

10. Shinba T, Takahashi K, Kanetaka S, Nedachi T, Yamaneki M, et al. (2012) A pilot study on electric field therapy for chronic pain with no obvious underlying diseases (in Japanese). Soc Integrative Med Jpn 5: 68-72.

11. Yanamoto H, Nakajo Y, Kataoka H, Iihara K (2013) High voltage electric potentials to enhance brain-derived neurotrophic factor levels in the brain. Front Neurol Neurosci 32: 129-138. [Crossref]

12. Nakagawa-Yagi Y, Hara H, Fujimori T, Yamaguchi T, Midorikawa A, et al. (2014) Non-targeted human plasma metabolomics reveals the changes in oleoylethanolamide, a lipid-derived signaling molecule, by acute exposure of electric field. Integr $\mathrm{Mol} \mathrm{Med}$ 1: 29-37.

13. Nakagawa-Yagi Y, Hara H, Yoshida Y, Midorikawa A, Hara A (2015) Discovery of a novel effect of electric field exposure on human plasma beta-endorphin and interleukin-12 levels: Insight into mechanisms of pain alleviation and defense against infection by electric field therapy. Integr Mol Med 2: 200-204.

14. Nakagawa-Yagi Y, Hara H, Nakagawa F, Sato M, Hara A (2016) Acute exposure to an electric field induces changes in human plasma 9-HODE, 13-HODE, and immunoreactive substance P levels: Insight into the molecular mechanisms of electric field therapy. Integr Mol Med 3: 600-605.

15. Nakagawa-Yagi Y, Hara H, Tsuboi H, Abe J, Hara A (2016) Effect of 3-hydroxybutyrate, an endogenous histone deacetylase inhibitor, on FOXO3A mRNA expression in human epithelial colorectal Caco-2 cells: Insight into the epigenetic mechanisms of electric field therapy. Integr Mol Med 3: 764-768.

16. Nakagawa-Yagi Y, Hara H, Nakanishi H, Tasaka T, Hara A (2017) Acute exposure to an electric field induces changes in human plasma lysophosphatidylcholine (lysoPC)-22:4 levels: Molecular insight into the docking of lysoPC-22:4 interaction with TRPV2. Integr Mol Med 4:1-7.

17. Ohtsuki T, Nabeta T, Nakanishi H, Kawahata H, Ogihara T, et al. (2017) Electric field exposure improves subjective symptoms related to sleeplessness in college students: A pilot study of electric field therapy for sleep disorder. Immunol Endocrine Metabolic agents Medicinal Chem 17: in press.

18. Hansen HS, Rosenkilde MM, Holst JJ, Schwartz TW (2012) GPR119 as a fat sensor. Trends Pharmacol Sci 33: 374-381. [Crossref]

19. Imae R, Inoue T, Nakasaki Y, Uchida Y, Ohba Y, et al. (2012) LYCAT, a homologue of C. elegans acl-8, acl-9, and acl-10, determines the fatty acid composition of phosphatidylinositol in mice. J Lipid Res 53: 335-347. [Crossref]

20. Baba T, Kashiwagi Y, Arimitsu N, Kogure T, Edo A, et al. (2014) Phosphatidic acid (PA)-preferring phospholipase A1 regulates mitochondrial dynamics. J Biol Chem 289: 11497-11511. [Crossref]

21. Bligh EG, Dyer WJ (1959) A rapid method of total lipid extraction and purification Canadian J Biochem Physiol 37: 911-917. [Crossref]

22. Engelstoft MS, Norn C, Hauge M, Holliday ND, Elster L, et al. (2014) Structural basis for constitutive activity and agonist-induced activation of the enteroendocrine fat sensor GPR119. Br J Pharmacol 171: 5774-5789. [Crossref]

23. Norn C, Hauge M, Engelstoft MS, Kim SH, Lehmann J, et al. (2015) MutationGuided Unbiased Modeling of the Fat Sensor GPR119 for High-Yield Agonist Screening. Structure 23: 2377-2386. [Crossref] 
Nakagawa-Yagi Y (2017) Molecular insight into the docking of lysophosphatidylethanolamine (lysoPE)-22:6 interaction with GPR119: Acute exposure to an electric field induces changes in human plasma lysoPE-22:6 and lysoPE-20:4 levels

24. Trott O, Olson AJ (2010) AutoDock Vina: improving the speed and accuracy of docking with a new scoring function, efficient optimization, and multithreading. $J$ Comput Chem 31: 455-461. [Crossref]

25. Hansen KB, Rosenkilde MM, Knop FK, Wellner N, Diep TA, et al. (2011) 2-Oleoyl glycerol is a GPR119 agonist and signals GLP-1 release in humans. J Clin Endocrinol Metab 96: E1409-1417. [Crossref]

26. Lan H, Lin HV, Wang CF, Wright MJ, Xu S, et al. (2012) Agonists at GPR119 mediate secretion of GLP-1 from mouse enteroendocrine cells through glucose-independent pathways. BrJ Pharmacol 165: 2799-2807. [Crossref]

27. Thuren T, Tulkki AP, Virtanen JA, Kinnunen PK (1987) Triggering of the activity of phospholipase A2 by an electric field. Biochemistry 26: 4907-4910. [Crossref]

28. Balsinde J, Balboa MA (2005) Cellular regulation and proposed biological functions of group VIA calcium-independet phospholipase A2 in activated cells. Cell Signal 17: 1052-1062. [Crossref]

29. Von Allmen CE, Schmitz N, Bauer M, Hinton HJ, Kurrer MO, et al. (2009) Secretory phospholipase A2-IID is an effector molecule of $\mathrm{CD}+\mathrm{CD} 25+$ regulatory T cells. Proc Natl Acd Sci USA 106: 11673-11678.

30. Wyss-Coray T, Rogers J (2012) Inflammation in Alzheimer disease - A brief review of the basic science and clinical literature. Cold Spring Harb Perspec Med 2: a006346. [Crossref]

31. Murakami M, Sato H, Miki Y, Yamamoto K, Taketomi Y (2015) A new era of secreted phospholipase $\mathrm{A}_{2}$, . J Lipid Res 56: 1248-1261. [Crossref]

32. Straub RH, Schradin C (2016) Chronic inflammatory systemic diseases: an evolutionary trade-off between acutely beneficial but chronically harmful programs. Evolution Med Public Health 2016:37-51. [Crossref]
33. Nakagawa-Yagi Y, Sato Y, Matsumoto E, Nakatsuka S, Sakaki T, et al. (2012) Pharmacological modulation of histone demethylase activity by a small molecule isolated from subcritical water extracts of Sasa senanensis leaves prolongs the lifespan of Drosophila melanogaster. BMC Complement Altern Med 12: 101. [Crossref]

34. Azam SS, Abbasi SW (2013) Molecular docking studies for the identification of novel melatoninergic inhibitors for acetylserotonin-O-methyltransferase using different docking routines. Theoretical Biol Medical Modelling 10: 63. [Crossref]

35. Tiruveedhula P, Mutagi AP, Paul K (2014) Insilco studies on membrane receptors of type 2 diabetes milletus. World J Pharmacy Pharmaceutical Sci 3: 1069-1096. [Crossref]

36. Lauffer LM, Iakoubov R, Brubaker PL (2009) GPR119 is essential for oleoylethanolamide-induced glucagon-like peptide-1 secretion from the intestinal enteroendocrine L-cell. Diabetes 58: 1058-1066. [Crossref]

37. Hansen HS, Rosenkilde MM, Holst JJ, Schwartz TW (2012) GPR119 as a fat sensor. Trends Pharmacol Sci 33: 374-381. [Crossref]

38. Cabou C, Burcelin R (2011) GLP-1, the gut-brain, and brain-periphery axes. Rev Diabet Stud 8: 418-431. [Crossref]

39. Furness JB, Rivera LR, Cho HJ, Bravo DM, Callaghan B (2013) The gut as a sensory organ. Nat Rev Gastroenterol Hepatol 10: 729-740. [Crossref]

40. Campolongo P, Roozendaal B, Trezza V, Cuomo V, Astarita G, et al. (2009) Fat-induced satiety factor oleoylethanolamide enhances memory consolidation. Proc Natl Acad Sci USA 106: 8027-8031. [Crossref]

41. Yanamoto H, Miyamoto S, Nakajo Y, Nakano Y, Hori T, et al. (2008) Repeated application of an electric field increases BDNF in the brain, enhances spatial learning, and induces infarct tolerance. Brain Res 1212: 79-88. [Crossref]

Copyright: (C2017 Nakagawa-Yagi Y. This is an open-access article distributed under the terms of the Creative Commons Attribution License, which permits unrestricted use, distribution, and reproduction in any medium, provided the original author and source are credited. 